\title{
Balancing BMI-rethinking the relationships between obesity, ageing and risk of dementia
}

A new large retrospective cohort study, published in The Lancet Diabetes and Endocrinology, has revealed a surprising association between being underweight in mid-life and latelife, and increased risk of dementia. These findings seem to be in stark contrast with the widely accepted hypothesis that being overweight or having obesity is linked to dementia later in life.

The researchers from the London School of Hygiene and Tropical Medicine, UK, initially assessed data from published studies that were identified in a large systematic analysis. However, on the basis of these reports having only small numbers of participants and few events, as well as a lack of information for some confounders, such as smoking and blood pressure, they concluded that much of the data from these studies were unreliable.

Data analysed in the new study comprised routine primary care data collected from patients in the UK between 1987 and 2013, which were obtained from the Clinical Practice Research Datalink (CPRD) and represents $~ 9 \%$ of the UK population. Patient information included physiological measurements, diagnoses, lifestyle information and referrals to secondary care. The authors believe that this study represents the largest study performed to-date of associations between BMI and risk of dementia, and is the first study with adequate statistical power to provide reliable estimates for both sexes over a wide age range.

Initially, data for 6,099,128 individuals aged $\geq 40$ years between 1992 and 2007 were selected from the CPRD, for which valid BMI data was available for 2,944,587 individuals. Standard definitions of BMI were implemented: underweight $<20 \mathrm{~kg} / \mathrm{m}^{2}$, healthy weight $20.0-24.9 \mathrm{~kg} / \mathrm{m}^{2}$, overweight $25.0-29.9 \mathrm{~kg} / \mathrm{m}^{2}$ and obese $\geq 30 \mathrm{~kg} / \mathrm{m}^{2}$. The data set was further filtered to exclude individuals without at least 12 months of historical data and to only include those with a weight $\geq 20 \mathrm{~kg}$, height of $121-214 \mathrm{~cm}$ and BMI of $15-50 \mathrm{~kg} / \mathrm{m}^{2}$. Finally, any patient with a history of dementia (Alzheimer disease, Lewy body disease, Pick's disease or dementia recorded at death) was excluded from the data set, which resulted in a study population of 1,958,191 individuals.

Poisson regression models were used to calculate incidence rates and rate ratios to determine the associations between BMI and risk of dementia. The analysis was adjusted for sex and for age at risk in 5-year bands, with the age bands being updated appropriately over time. The adjusted models were then fitted to control for possible confounding variables, such as smoking, alcohol consumption, use of statins and antihypertensives, having diabetes mellitus and previous myocardial infarction.

Analysis of the distribution of BMI measurements by age and sex revealed

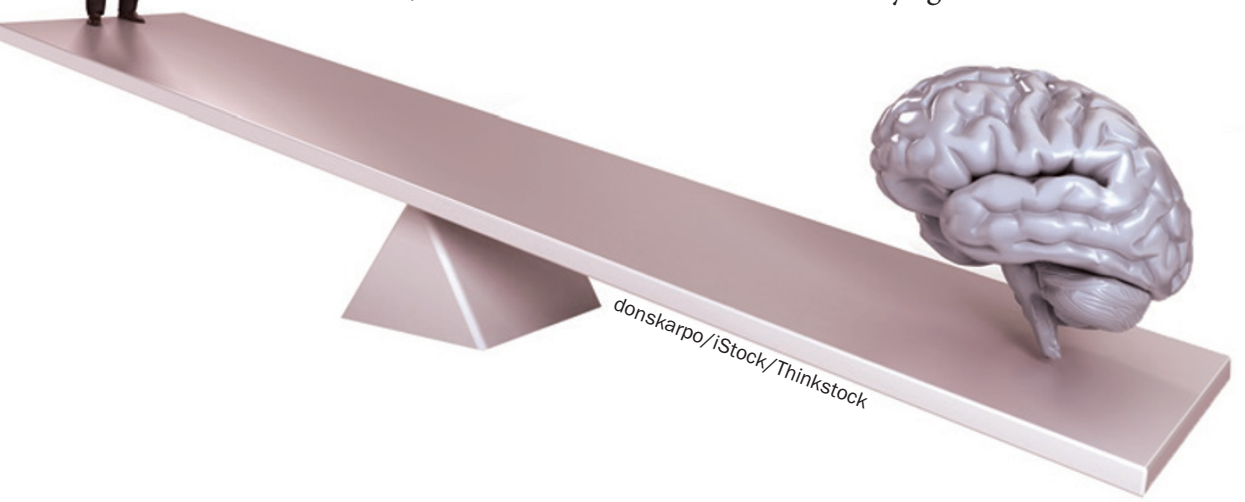

that obesity was more prevalent in individuals at younger rather than older ages; prevalence of underweight was increased in women compared with men, but increased with age in both sexes. Dementia was also strongly associated with increasing age, and, in those aged $>70$ years, dementia was more prevalent in women than in men.

Estimation of age-adjusted and sexadjusted rates of dementia showed that increasing BMI measurements were inversely associated with risk of dementia. Individuals who were underweight had a $64 \%$ higher risk of dementia than individuals with overweight (median BMI $\sim 26-27 \mathrm{~kg} / \mathrm{m}^{2}$ ). Additionally, the cumulative risk of dementia over time was highest for those who were underweight, followed by individuals of a healthy weight. However, participants with overweight or obesity showed a reduced cumulative risk of dementia with increasing age. Furthermore, the increased risk persisted for as long as 15 years following an underweight BMI recording.

The findings from this study are controversial, as they contradict previously published reports of positive associations of BMI with risk of dementia and those that have shown no associations. The authors suggest that the inconsistencies in the field might be due to the small size of these studies and that studies with long follow-up periods could help to clarify the validity of these associations. Furthermore, this study does have some limitations, such as potential selection bias and a possible underdiagnosis of dementia in the CPRD, which will need to be addressed in future investigations.

Jennifer Sargent 